\title{
Bi-maximal Neutrino Mixing And Anomalous Flavor $\mathcal{U}(1)$
}

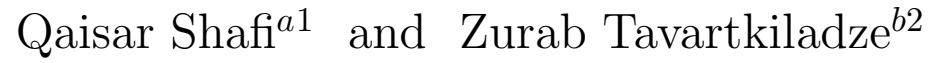 \\ ${ }^{a}$ Bartol Research Institute, University of Delaware, Newark, DE 19716, USA \\ ${ }^{b}$ Institute of Physics, Georgian Academy of Sciences, 380077 Tbilisi, Georgia
}

\begin{abstract}
We describe how bi-maximal neutrino mixing can be realized in realistic models based on MSSM and SUSY GUTs such as $S U(5)$ and $S O(10)$. A crucial role is played by an anomalous $\mathcal{U}(1)$ flavor symmetry, which also helps understand the observed charged fermion mass hierarchies and the magnitudes of the CKM matrix elements. While in MSSM a variety of solutions for the solar neutrino puzzle are possible, SUSY $S U(5)$ and $S O(10)$ only permit the large mixing angle MSW solution. Models in which the $\mathcal{U}(1)$ symmetry also mediates SUSY breaking allow both the large mixing angle and the low MSW solutions.

We also present renormalization group studies for the neutrino mass matrix, generated through the $\mathcal{U}(1)$ flavor symmetry. Our analysis shows that renormalization does not change the desirable picture of bi-maximal mixing.
\end{abstract}

\footnotetext{
${ }^{1}$ E-mail address: shafi@bartol.udel.edu

${ }^{2}$ E-mail address: z_tavart@osgf.ge
} 
Despite its remarkable experimental successes there is little doubt that the standard model based on $S U(3) \times S U(2) \times U(1)$ must be part of a more complete theory. Support for this statement comes from a variety of sources. Two that are particularly relevant for us here are the atmospheric (and solar) neutrino puzzles, as well as the well known flavor problem. We will attempt to resolve them within a unified framework such as provided by the minimal supersymmetric standard model (MSSM), $S U(5)$ or $S O(10)$, supplemented by a flavor $\mathcal{U}(1)$ symmetry.

In the charged fermion sector there are noticeable hierarchies within the charged fermion Yukawa couplings and the CKM matrix elements. Since the mass of the top quark is close to the electroweak symmetry breaking scale $(\sim 100 \mathrm{GeV})$, its Yukawa coupling is of order unity $\left(\lambda_{t} \sim 1\right)$. As far as the Yukawa couplings of the $b$ quark and $\tau$ lepton are concerned, their values could vary in a range $\lambda_{b} \sim \lambda_{\tau} \sim 10^{-2}-1$, depending on the value of the MSSM parameter $\tan \beta(\sim 1-60)$. Introducing the dimensionless parameter $\epsilon \simeq 0.2$ (close to the Wolfenstein parameter $\lambda$ ), one can express the observed hierarchies between the charged fermion Yukawa couplings as follows:

$$
\begin{gathered}
\lambda_{t} \sim 1, \quad \lambda_{u}: \lambda_{c}: \lambda_{t} \sim \epsilon^{6}: \epsilon^{3}: 1, \\
\lambda_{b} \sim \lambda_{\tau} \sim \lambda_{t} \frac{m_{b}}{m_{t}} \tan \beta, \quad \lambda_{d}: \lambda_{s}: \lambda_{b} \sim \epsilon^{5}: \epsilon^{2}: 1, \\
\lambda_{e}: \lambda_{\mu}: \lambda_{\tau} \sim \epsilon^{5}: \epsilon^{2}: 1,
\end{gathered}
$$

while for the CKM matrix elements:

$$
V_{u s} \sim \epsilon, \quad V_{c b} \sim \epsilon^{2}, \quad V_{u b} \sim \epsilon^{3} .
$$

In constructing models, one should arrange for a natural understanding of the hierarchies in (11)-(雨).

The latest atmospheric and solar neutrino data (see [1] and [2] respectively) seem to provide convincing confidence in the phenomena of neutrino oscillations. Ignoring the LSND data [3], the atmospheric and solar anomalies can be explained within the three states of active neutrinos. In this paper we will study oscillation scenarios without the sterile neutrinos, which in any case maybe disfavored by the data [2, 1, 4].

The atmospheric neutrino data suggest oscillations of $\nu_{\mu}$ into $\nu_{\tau}$, with the following oscillation parameters:

$$
\begin{gathered}
\mathcal{A}\left(\nu_{\mu} \rightarrow \nu_{\tau}\right) \equiv \sin ^{2} 2 \theta_{\mu \tau} \simeq 1 \\
\Delta m_{\mathrm{atm}}^{2} \simeq 3 \cdot 10^{-3} \mathrm{eV}^{2} .
\end{gathered}
$$


The solar neutrino anomaly seems consistent with a variety of oscillation scenarios, amongst which the most likely seems to be large angle MSW (LAMSW) oscillation of $\nu_{e}$ into $\nu_{\mu, \tau}$ [2], with the oscillation parameters:

$$
\begin{gathered}
\mathcal{A}\left(\nu_{e} \rightarrow \nu_{\mu, \tau}\right) \equiv \sin ^{2} 2 \theta_{e \mu, \tau} \simeq 0.8, \\
\Delta m_{\mathrm{sol}}^{2} \simeq 2 \cdot 10^{-5} \mathrm{eV}^{2} .
\end{gathered}
$$

The scenario of low MSW (LOW MSW) oscillations of solar neutrinos require:

$$
\begin{gathered}
\sin ^{2} 2 \theta_{e \mu, \tau} \simeq 1.0, \\
\Delta m_{\mathrm{sol}}^{2} \simeq 8 \cdot 10^{-8} \mathrm{eV}^{2},
\end{gathered}
$$

while the small angle MSW (SA MSW) oscillations are realized with:

$$
\begin{gathered}
\sin ^{2} 2 \theta_{e \mu, \tau} \simeq 5 \cdot 10^{-3}, \\
\Delta m_{\mathrm{sol}}^{2} \simeq 5 \cdot 10^{-6} \mathrm{eV}^{2} .
\end{gathered}
$$

Finally, the large angle vacuum oscillation (LAVO) solution requires

$$
\begin{gathered}
\sin ^{2} 2 \theta_{e \mu, \tau} \simeq 0.7, \\
\Delta m_{\mathrm{sol}}^{2} \simeq 8 \cdot 10^{-11} \mathrm{eV}^{2} .
\end{gathered}
$$

It is worth noting that within MSSM, the neutrinos acquire masses only through non-renormalizable $d=5$ Planck scale operators $l_{i} l_{j} h_{u}^{2} / M_{P}$ which, for $\left\langle h_{u}^{0}\right\rangle \sim 100 \mathrm{GeV}$, $M_{P}=2.4 \cdot 10^{18} \mathrm{GeV}$ (reduced Planck mass) give $m_{\nu_{i}} \sim 10^{-5} \mathrm{eV}$. Therefore, already for atmospheric data (5) [and also for solar neutrino anomalies with (6)-(8)] we need physics beyond the MSSM. In order to generate the appropriate neutrino masses, we will introduce heavy right handed neutrino states $\mathcal{N}_{i}$. Then, the 'light' left handed neutrinos will acquire masses through the see-saw mechanism [5].

In building neutrino oscillation scenarios, the main challenge is to generate desirable magnitudes for neutrino masses and their mixings. And to understand why in some cases, the mixing angles are large (and even maximal), while the quark CKM matrix elements (4) are suppressed. Below we will present a mechanism which successfully resolves all of these problems.

Before proceeding to the model, let us study a specific neutrino mass matrix texture, which provides bi-maximal neutrino mixing. Consider the mass matrix for three active neutrino flavors: 


$$
\hat{M}_{\nu}=\left(\begin{array}{ccc}
0 & m_{1} & m_{2} \\
m_{1} & 0 & 0 \\
m_{2} & 0 & 0
\end{array}\right)
$$

and assume that

$$
m_{1} \sim m_{2}
$$

are real mass parameters. Performing the transformation $U_{1}^{T} M_{\nu} U_{1} \equiv M_{\nu}^{\prime}$, where

$$
\begin{gathered}
U_{1}=\left(\begin{array}{ccc}
1 & 0 & 0 \\
0 & c_{\theta} & -s_{\theta} \\
0 & s_{\theta} & c_{\theta}
\end{array}\right), \\
s_{\theta} \equiv \sin \theta, \quad c_{\theta} \equiv \cos \theta, \quad \tan \theta=\frac{m_{2}}{m_{1}},
\end{gathered}
$$

the mass matrix acquires the off-diagonal form:

$$
\hat{M}^{\prime}{ }_{\nu}=\left(\begin{array}{ccc}
0 & m & 0 \\
m & 0 & 0 \\
0 & 0 & 0
\end{array}\right), \quad m=\sqrt{m_{1}^{2}+m_{2}^{2}},
$$

which is diagonalized through transformations with maximal rotating angles:

$$
\begin{gathered}
U_{2}^{T} \hat{M}^{\prime}{ }_{\nu} U_{2} \equiv \hat{M}_{\nu}^{\text {diag }}=\operatorname{Diag}(m,-m, 0) \\
U_{2}=\left(\begin{array}{ccc}
\frac{1}{\sqrt{2}} & -\frac{1}{\sqrt{2}} & 0 \\
\frac{1}{\sqrt{2}} & \frac{1}{\sqrt{2}} & 0 \\
0 & 0 & 1
\end{array}\right) .
\end{gathered}
$$

The rotation matrix which connects the mass eigenstate basis to the flavor one is given by:

$$
U_{\nu}=U_{1} U_{2}=\left(\begin{array}{ccc}
\frac{1}{\sqrt{2}} & -\frac{1}{\sqrt{2}} & 0 \\
\frac{1}{\sqrt{2}} c_{\theta} & \frac{1}{\sqrt{2}} c_{\theta} & -s_{\theta} \\
\frac{1}{\sqrt{2}} s_{\theta} & \frac{1}{\sqrt{2}} s_{\theta} & c_{\theta}
\end{array}\right),
$$

which is precisely a bi-maximal mixing matrix. Through (17), using the expression for the oscillation amplitude 


$$
\mathcal{A}\left(\nu_{\alpha} \rightarrow \nu_{\beta}\right)=4 \Sigma_{j<i} U_{\nu}^{\alpha j} U_{\nu}^{\alpha i} U_{\nu}^{\beta j} U_{\nu}^{\beta i},
$$

( $\alpha, \beta$ denote flavor indices and $i, j$ the mass eigenstates), the oscillation amplitudes for the atmospheric and solar neutrinos are respectively

$$
\begin{gathered}
\mathcal{A}\left(\nu_{\mu} \rightarrow \nu_{\tau}\right) \equiv \sin ^{2} 2 \theta_{\mu \tau}=\frac{4 m_{1}^{2} m_{2}^{2}}{\left(m_{1}^{2}+m_{2}^{2}\right)^{2}} \sim 1, \\
\mathcal{A}\left(\nu_{e} \rightarrow \nu_{\mu, \tau}\right) \equiv \sin ^{2} 2 \theta_{e \mu, \tau}=1 .
\end{gathered}
$$

In (19) we have taken into account (11), while the second equation in (20) holds without any assumptions. For $m_{1} \simeq m_{2}$ we will have $\sin ^{2} 2 \theta_{\mu \tau} \simeq 1$, which means that the second mass eigenstate is $\nu_{2} \simeq \frac{1}{\sqrt{2}}\left(\nu_{\mu}+\nu_{\tau}\right)$, and therefore the $\nu_{e}$ state should oscillate with $50 \%$ probability each into $\nu_{\mu}$ and $\nu_{\tau}$. However, at this level $\nu_{e}-\nu_{2}$ oscillation cannot occur because of zero mass squared difference. Indeed, the neutrino mass spectra is

$$
m_{\nu_{1}}=m_{\nu_{2}}=m, \quad m_{\nu_{3}}=0
$$

(inverted hierarchy), and consequently:

$$
\Delta m_{32}^{2}=m^{2}, \quad \Delta m_{21}^{2}=0 .
$$

From (5) we have

$$
m^{2} \sim m_{\mathrm{atm}}^{2} \sim 10^{-3} \mathrm{eV}^{2} .
$$

In order to get a nonzero $\Delta m_{21}^{2}$, the texture (10) should be slightly modified:

$$
\hat{M}_{\nu}=\left(\begin{array}{ccc}
\rho_{1} & m_{1} & m_{2} \\
m_{1} & \rho_{2} & \rho_{4} \\
m_{2} & \rho_{4} & \rho_{3}
\end{array}\right),
$$

with small deviations $\rho_{i} \ll m_{1}, m_{2}$, in order to preserve bi-maximal mixing. Of course, it is possible to choose $\rho_{i}$ s by hand in such a way as to get the desirable picture. However, it would be much nicer to provide a natural mechanism for realizing this. The goal is therefore to construct a model that will allow us to estimate values of $\rho_{i}$, which will give nonzero $\Delta m_{12}^{2}$, thereby guaranteeing $\nu_{e}-\nu_{2}$ oscillations. The value of $\Delta m_{12}^{2}$ will select one of the currently viable oscillation scenarios for solar neutrinos. In [6], the texture (10) was obtained by imposing $L_{e}-L_{\mu}-L_{\tau}$ symmetry. Refs. [7] presented models in which non-zero $\rho_{i}$ emerge at the two loop level and the large angle vacuum oscillation scenario is realized. In [8] a $\mathcal{U}(1)$ flavor symmetry is used for providing this bi-maximal mixing 
matrix. In this paper we will follow the strategy of [8]. Let us finally note that the texture in (10) is also discussed in [9].

We introduce a $\mathcal{U}(1)$ flavor symmetry, which distinguishes the families through the prescription of $\mathcal{U}(1)$ charges. In many cases it turns out that the $\mathcal{U}(1)$ is anomalous. It is well known that such anomalous $\mathcal{U}(1)$ factors can arise in string theories. Cancellation of the anomaly occurs through the Green-Schwarz mechanism [10]. Due to the anomaly the Fayet-Illiopoulos D-term $\xi \int d^{4} \theta V_{A}$ is always generated, where in string theory $\xi$ is given by 11

$$
\xi=\frac{g_{A}^{2} M_{P}^{2}}{192 \pi^{2}} \operatorname{Tr} Q
$$

The $D_{A}$-term will have the form:

$$
\frac{g_{A}^{2}}{8} D_{A}^{2}=\frac{g_{A}^{2}}{8}\left(\Sigma Q_{a}\left|\varphi_{a}\right|^{2}+\xi\right)^{2}
$$

where $Q_{a}$ is the 'anomalous' charge of $\varphi_{a}$ superfield. For $\mathcal{U}(1)$ breaking we introduce the singlet superfield $X$ with $\mathcal{U}(1)$ charge $Q_{X}$. Assuming $\xi>0[\operatorname{Tr} Q>0$ in (25)], and taking

$$
Q_{X}=-1
$$

the cancellation of $D_{A}$ in (26) and nonzero $\langle X\rangle$ are ensured: $\langle X\rangle=\sqrt{\xi}$. Further, we will take

$$
\frac{\langle X\rangle}{M_{P}} \equiv \epsilon \simeq 0.2
$$

where $\epsilon$ turns out to be an important expansion parameter. Let us note that an anomalous $\mathcal{U}(1)$ for understanding the hierarchies of fermion masses and mixings has been discussed in several papers of [12]. In [13] a variety of neutrino oscillation scenarios were constructed with the help of $\mathcal{U}(1)$.

Starting our investigation with the neutrino sector, let us first discuss two ways of obtaining large/maximal neutrino mixings with the help of $\mathcal{U}(1)$ flavor symmetry. With two flavors of lepton doublets $l_{1}$ and $l_{2}$, one way of having large mixing is the so-called democratic approach. In this case the $\mathcal{U}(1)$ symmetry does not distinguish the two flavors [14, i.e. they have the same $\mathcal{U}(1)$ charges $Q_{l_{1}}=Q_{l_{2}}=n$ (positive integer number). In this case, the expected neutrino mass matrix will be:

$$
\hat{m}_{\nu}=\left(\begin{array}{ll}
1 & 1 \\
1 & 1
\end{array}\right) \bar{m} \epsilon^{2 n}, \quad \bar{m}=\frac{h_{u}^{2}}{M}
$$


with entries of order unity $\left(\bar{M}\right.$ is some mass scale and we have assumed $\left.Q_{h_{u}}=0\right)$. Therefore, naturally large $\nu_{1}-\nu_{2}$ mixing is expected, $\sin ^{2} 2 \theta_{12} \sim 1$. Also, one can expect $m_{\nu_{1}} \sim m_{\nu_{2}}$, and if this mechanism is used for atmospheric neutrinos, somehow one has to keep one state light, in order to accomodate also the solar neutrino puzzle. This can be done [15, 16] by introducing a single right handed neutrino $\mathcal{N}$. After integrating it out due to degeneracy, only one state acquires mass. The remaining states can be used for the solar neutrino puzzle. An appropriate mass scale for the latter can be generated by introducing a relatively heavy right handed state $\mathcal{N}^{\prime}$ with suppressed coupling with $\mathcal{N}$.

A different approach is the so-called maximal mixing mechanism [17]. It is realized by assigning different $\mathcal{U}(1)$ charges for the flavors $l_{1}, l_{2}$. Introducing two right handed states $\mathcal{N}_{1}, \mathcal{N}_{2}$ and the following $\mathcal{U}(1)$ charge prescriptions

$$
\begin{gathered}
Q_{l_{1}}=k+n, \quad Q_{l_{2}}=k, \quad Q_{h_{u}}=0, \\
Q_{\mathcal{N}_{1}}=-Q_{\mathcal{N}_{2}}=k+k^{\prime},
\end{gathered}
$$

with $k, n, k^{\prime}>0, n \geq k^{\prime}$, the 'Dirac' and 'Majorana' couplings will have the forms:

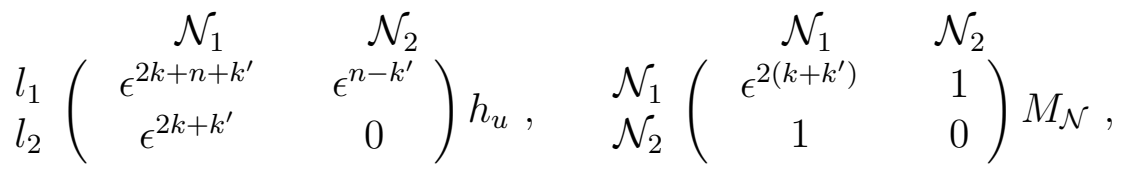

After integrating out the heavy $\mathcal{N}_{1}, \mathcal{N}_{2}$ states, the neutrino mass matrix is given by

$$
\hat{m}_{\nu}=\left(\begin{array}{cc}
\epsilon^{n} & 1 \\
1 & 0
\end{array}\right) \bar{m}, \quad \bar{m}=\frac{h_{u}^{2} \epsilon^{2 k+n}}{M_{\mathcal{N}}}
$$

a quasi off-diagonal form, leading to a mixing angle

$$
\sin ^{2} 2 \theta_{12}=1-\mathcal{O}\left(\epsilon^{2 n}\right),
$$

which is close to maximal mixing. The form (32) is guaranteed by the appropriate zero entries in (31), which are ensured by $\mathcal{U}(1)$ symmetry. This mechanism turns out to be very convenient for achieving nearly maximal mixings between neutrino flavors within various realistic models, such as $S U(5)$ [16], $S O(10)$ [18], $S U(4)_{c} \times S U(2)_{L} \times S U(2)_{R}$ [19], etc.

Returning to our scheme, we attempt to obtain the bi-maximal texture (10) through $\mathcal{U}(1)$ flavor symmetry. For this, we will combine the two mechanisms for maximal mixing discussed above. Namely, the second and third lepton doublet states will have the same $\mathcal{U}(1)$ charges, which will lead to their large mixing. The state $l_{1}$ will have a suitable charge, one that ensures maximal $\nu_{1}-\nu_{2}$ mixing. 
Introducing two right handed $\mathcal{N}_{1,2}$ neutrino states and choosing $\mathcal{U}(1)$ charges as

$$
\begin{gathered}
Q_{X}=-1, Q_{l_{2}}=Q_{l_{3}}=k, Q_{l_{1}}=k+n, Q_{h_{u}}=Q_{h_{d}}=0 \\
Q_{\mathcal{N}_{1}}=-Q_{\mathcal{N}_{2}}=k+k^{\prime}
\end{gathered}
$$

with

$$
k, n, k^{\prime}>0, \quad n \geq k^{\prime},
$$

the 'Dirac' and 'Majorana' couplings will have forms:

$$
\begin{array}{cc}
\mathcal{N}_{1} & \mathcal{N}_{2} \\
l_{1} \\
l_{2} \\
l_{3}
\end{array}\left(\begin{array}{cc}
\epsilon^{2 k+n+k^{\prime}} & \epsilon^{n-k^{\prime}} \\
\epsilon^{2 k+k^{\prime}} & 0 \\
\epsilon^{2 k+k^{\prime}} & 0
\end{array}\right) h_{u}, \quad \mathcal{N}_{1}\left(\begin{array}{cc}
\mathcal{N}_{1} & \mathcal{N}_{2} \\
\epsilon^{2\left(k+k^{\prime}\right)} & 1 \\
1 & 0
\end{array}\right) M_{\mathcal{N}}
$$

After integrating out $\mathcal{N}_{1,2}$, we obtain the texture

$$
\hat{M}_{\nu} \propto\left(\begin{array}{ccc}
\epsilon^{n} & 1 & 1 \\
1 & 0 & 0 \\
1 & 0 & 0
\end{array}\right) m, \quad m=\frac{\epsilon^{2 k+n} h_{u}^{2}}{M_{\mathcal{N}}},
$$

which differs from (10) by a non-zero $(1,1)$ element. In (37) coefficients of order unity are assumed. The nonzero $(1,1)$ entry in (37) guarantees that $\Delta m_{12}^{2} \neq 0$. Using (18) and (37) the oscillation parameters are:

$$
\begin{gathered}
\Delta m_{32}^{2} \equiv m_{\mathrm{atm}}^{2}=m^{2} \sim 10^{-3} \mathrm{eV}^{2}, \\
\mathcal{A}\left(\nu_{\mu} \rightarrow \nu_{\tau}\right) \sim 1, \\
\Delta m_{21}^{2} \simeq 2 m_{\mathrm{atm}}^{2} \epsilon^{n}, \\
\mathcal{A}\left(\nu_{e} \rightarrow \nu_{\mu, \tau}\right)=1-\mathcal{O}\left(\epsilon^{2 n}\right) .
\end{gathered}
$$

Note, that the model does not constrain $n$ for the time being. So, LAMSW, LOW MSW and LAVO solutions for solar neutrinos, can be realized. With prescription (34), the expected contribution from the charged lepton sector to the angles $\theta_{23}^{l}$ and $\theta_{12}^{l}$ will be $\sim 1$ and $\sim \epsilon^{n}$ respectively. These do not change the form of $V_{\nu}$ in (17).

The $\mathcal{U}(1)$ charge selection in (34) nicely blends with the charged fermion sector. Indeed, considering the following prescription: 


$$
\begin{gathered}
Q_{q_{3}}=0, \quad Q_{q_{2}}=2, \quad Q_{q_{1}}=3, \quad Q_{d_{3}^{c}}=Q_{d_{2}^{c}}=p+k, \\
Q_{d_{1}^{c}}=p+k+2, \quad Q_{u_{3}^{c}}=0, \quad Q_{u_{2}^{c}}=1, \quad Q_{u_{1}^{c}}=3, \\
Q_{e_{3}^{c}}=p, \quad Q_{e_{2}^{c}}=p+2, \quad Q_{e_{1}^{c}}=p+5-n,
\end{gathered}
$$

the structures of Yukawa matrices, for up-down quarks and charged leptons respectively:

$$
\begin{gathered}
q_{1}\left(\begin{array}{ccc}
u_{1}^{c} & u_{2}^{c} & u_{3}^{c} \\
\epsilon^{6} & \epsilon^{4} & \epsilon^{3} \\
\epsilon^{5} & \epsilon^{3} & \epsilon^{2} \\
\epsilon^{3} & \epsilon & 1
\end{array}\right) h_{u}, \\
q_{3} \\
q_{1}\left(\begin{array}{ccc}
d_{1}^{c} & d_{2}^{c} & d_{3}^{c} \\
\epsilon^{5} & \epsilon^{3} & \epsilon^{3} \\
\epsilon^{4} & \epsilon^{2} & \epsilon^{2} \\
\epsilon^{2} & 1 & 1
\end{array}\right) \epsilon^{p+k} h_{d}, \\
q_{3} \\
l_{1}\left(\begin{array}{ccc}
e_{1}^{c} & e_{2}^{c} & e_{3}^{c} \\
l_{2} & \epsilon^{n+2} & \epsilon^{n} \\
\epsilon_{3} & \epsilon^{5-n} & 1 \\
\epsilon^{5-n} & \epsilon^{2} & 1
\end{array}\right) \epsilon^{p+k} h_{d} .
\end{gathered}
$$

Upon diagonalization of (41)-(43) it is easy to verify that the desired relations (11)-(41) for the Yukawa couplings and CKM matrix elements are realized. From (42), (43) we have

$$
\tan \beta \sim \epsilon^{p+k} \frac{m_{t}}{m_{b}} .
$$

As we previously mentioned, MSSM does not fix the values of $n, k, p$ in (34), (40). Because of this, the solar neutrino oscillation scenario is not specified. According to (39) there is possibility for all three: LAMSW, LOW MSW and LAVO solutions. Namely, for $n=3$ we have $\Delta m_{12}^{2} \sim 10^{-5} \mathrm{eV}^{2}$, which corresponds to LAMSW. $n=6$ give $\Delta m_{12}^{2} \sim 10^{-7} \mathrm{eV}^{2}$, which is the scale for LOW MSW, while $n=10$ generates the scale $\Delta m_{12}^{2} \sim 10^{-10} \mathrm{eV}^{2}$, corresponding to LAVO solution. It would be interesting to look for models/scenarios, which somehow fix the values of $n, p, k$, that dictate the solar neutrino oscillation scenario, Here we present two cases in which this happens.

Consider SUSY SU(5) GUT. Its matter sector consists of $10+\overline{5}$ supermultiplets per generation. Due to these unified multiplets:

$$
Q_{q}=Q_{e^{c}}=Q_{u^{c}}=Q_{10}, \quad Q_{l}=Q_{d^{c}}=Q_{\overline{5}} .
$$


Hierarchies of the CKM matrix elements in (4) dictate the relative $\mathcal{U}(1)$ charges of the 10-plets

$$
Q_{10_{3}}=0, \quad Q_{10_{2}}=2, \quad Q_{10_{1}}=3,
$$

while the Yukawa hierarchies (1)-(司), together with (46), require that

$$
Q_{\overline{5}_{3}}=Q_{\overline{5}_{2}}=k, \quad Q_{\overline{5}_{1}}=k+2 .
$$

Comparing (45)-(47) with (34), (40) we see that the minimal $S U(5)$ GUT fixes $n$ and $p$ as

$$
n=2, \quad p=0 .
$$

The mass squared splitting in (39) then equals $\Delta m_{12}^{2} \sim 10^{-4} \mathrm{eV}^{2}$, which is a reasonable scale for LAMSW scenario. Therefore, realisation of our bi-maximal mixing scenario in the framework of $S U(5)$ GUT dictates that the LAMSW scenario is responsible for the solar neutrino deficit. The same conclusion can be reached for $S O(10)$ GUT where we have three 16-plets of the chiral supermultiplets which unify the quark-lepton superfields. We do not present the details here but refer the reader to [18], where an explicit $S O(10)$ model with anomalous $\mathcal{U}(1)$ flavor symmetry is considered for explanations of fermion masses, their mixings, as well as neutrino anomalies.

For our second example, we present a model in which anomalous flavor $\mathcal{U}(1)$ also mediates SUSY breaking [20]. In this case soft masses $\left(\sim m_{S}\right)$, emerging through nonzero $D_{A}$-term, dominate over $m_{3 / 2}$ (gravitino mass). This fact can be used for natural suppression of FCNC through the so-called decoupling solution [21]. The generated soft mass squared for the scalar component of superfield $\phi_{a}$ is

$$
m_{\tilde{\phi}_{i}}^{2}=m_{S}^{2} Q_{a} .
$$

Therefore, sparticles with non-zero $\mathcal{U}(1)$ charges will be relatively heavy. A scale $\sim 10 \mathrm{TeV}$ is enough for adequate suppression of all FCNC processes [22]. It turns out that in this scenario there also occurs suppression of dimension five nucleon decay [23, 24]. In 24] we presented a specific model of anomalous flavor $\mathcal{U}(1)$ mediated SUSY breaking. Suppressions of FCNC and $d=5$ nucleon decay were guaranteed through heavy ( $10 \mathrm{TeV}$ ) first and second sparticle generations (and also through heavy $\tilde{b}, \tilde{\tau}$, which require the low $\tan \beta$ regime).

In order to have possitive soft squared masses for squarks and sleptons, they should have charges $Q_{\tilde{q}, \tilde{l}}>0$ [see (49)]. On the other hand, from (39), the realization of LAVO requires $n=10$, which for $Q_{e^{c}}$ gives a negative $\mathcal{U}(1)$ charge [see (40). $p+k \leq 3$, since $\left.10^{-2} \lesssim \lambda_{b, \tau} \lesssim 1\right]$ and therefore negative $m_{\tilde{e}^{c}}^{2}$. However, the value $n=3$, which is needed 
for LAMSW, is possible. Also, the case $n=6$ is possible if $p \geq 1$, in order to guarantee $Q_{e^{c}} \geq 0$ in (40). This gives the LOW MSW solution. Note also, that in this particular case the value of $\tan \beta$ in (44) is either intermediate or low. Therefore, the scenario in which anomalous flavor $\mathcal{U}(1)$ mediates SUSY breaking permits LAMSW and LOW MSW oscillations for solar neutrinos, but excludes LAVO even in the framework of MSSM.

As we have seen the texture (37) provides bi-maximal neutrino mixing. However, $\hat{M}_{\nu}$ has the form (37) if we neglect renormalizations. In order for the analysis to be complete, we should take this into account. Crucial for this are the structures in (36), which are prescribed at scale $M_{X}\left(\mathcal{U}(1)\right.$ summetry breaking scale). In the ranges $M_{X}-M_{\mathcal{N}}$ and $M_{\mathcal{N}}-M_{Z}$, renormalization effects will occur and one has to make sure that the successful picture of bi-maximal mixing will not be spoiled. Let us now confirm that this is indeed the case here.

Between $M_{X}$ and $M_{\mathcal{N}}$ the states $\mathcal{N}_{1,2}$ are not decoupled and we have to renormalize the following couplings:

$$
l \hat{\lambda}_{\nu} \mathcal{N} h_{u}+\mathcal{N} \hat{M} \mathcal{N}
$$

Renormalization group equations (RGE) for the elements of $\hat{\lambda}_{\nu}$ and $\hat{M}$ respectively are:

$$
\begin{gathered}
16 \pi^{2} \frac{d \lambda_{\nu}^{i j}}{d t}=\left(-c_{a} g_{a}^{2} \lambda_{\nu}+2 \lambda_{\nu} \lambda_{\nu}^{T} \lambda_{\nu}+\lambda_{e} \lambda_{e}^{T} \lambda_{\nu}+3 \operatorname{tr}\left(\lambda_{u} \lambda_{u}^{T}\right) \lambda_{\nu}\right)^{i j} \\
16 \pi^{2} \frac{d}{d t} M^{i j}=\left(M \lambda_{\nu}^{T} \lambda_{\nu}+\lambda_{\nu}^{T} \lambda_{\nu} M\right)^{i j}
\end{gathered}
$$

where $t=\ln \mu$ is a renormalization scale factor and $g_{a}(a=1,2,3)$ are the gauge couplings of $U(1)_{Y}, S U(2)_{L}$ and $S U(3)_{c}$ respectively. For MSSM $c_{a}=\left(\frac{3}{5}, 3,0\right)$.

We will work in a basis in which $\hat{\lambda}_{e}$ is a diagonal. The lepton CKM matrix is then completely determined through the neutrino mixing matrix. In this basis, instead of (36), $\hat{\lambda}_{\nu}$ has the form

$$
\hat{\lambda}_{\nu}=\left(\begin{array}{cc}
\epsilon^{2 k+n+k^{\prime}} & \epsilon^{n-k^{\prime}} \\
\epsilon^{2 k+k^{\prime}} & \epsilon^{2 n-k^{\prime}} \\
\epsilon^{2 k+k^{\prime}} & \epsilon^{2 n-k^{\prime}}
\end{array}\right) .
$$

It is easy to see that the non-zero but suppressed $(2,2)$ and $(3,2)$ entries in (53) do not change results. The important thing is that, after renormalization,

$$
\delta_{2(3)} \equiv \frac{\lambda_{\nu}^{22(32)}}{\lambda_{\nu}^{12}} \lesssim \epsilon^{n} .
$$

Also, in the texture for $\mathcal{N}_{1,2}$ in $(36)$, the elements $M^{11}, M^{22}$ must satisfy 


$$
\Delta_{1(2)} \equiv \frac{M^{11(22)}}{M^{12}} \ll 1 .
$$

At scale $M_{X}$ the conditions (54), (55) are guaranteed by $\mathcal{U}(1)$, and we have to make sure that $\delta_{2}, \delta_{3}, \Delta_{1}, \Delta_{2}$ do not get significant contributions from renormalizations. From (51), (52) the RGEs for $\delta$ and $\Delta$, to a good approximation, are:

$$
\begin{aligned}
16 \pi^{2} \frac{d \delta_{2}}{d t} & \simeq\left(\lambda_{\mu}^{2}+4 \epsilon^{4 k+2 k^{\prime}}\right) \delta_{2}+2 \epsilon^{4 k+2 k^{\prime}+n}, \\
16 \pi^{2} \frac{d \delta_{3}}{d t} & \simeq\left(\lambda_{\tau}^{2}+4 \epsilon^{4 k+2 k^{\prime}}\right) \delta_{3}+2 \epsilon^{4 k+2 k^{\prime}+n}, \\
16 \pi^{2} \frac{d \Delta_{1}}{d t} & \simeq\left(2 \epsilon^{4 k+2 k^{\prime}}-\epsilon^{2 n-2 k^{\prime}}\right) \Delta_{1}+6 \epsilon^{2 k+2 n}, \\
16 \pi^{2} \frac{d \Delta_{2}}{d t} & \simeq\left(\epsilon^{2 n-2 k^{\prime}}-2 \epsilon^{4 k+2 k^{\prime}}\right) \Delta_{2}+6 \epsilon^{2 k+2 n} .
\end{aligned}
$$

The approximate solutions of (56)-(59) are

$$
\begin{gathered}
\delta_{2} \simeq \frac{1}{16 \pi^{2}}\left[\left(\lambda_{\mu}^{2}+4 \epsilon^{4 k+2 k^{\prime}}\right) \delta_{2}^{0}+2 \epsilon^{4 k+2 k^{\prime}+n}\right] \ln \frac{M_{\mathcal{N}}}{M_{X}}+\delta_{2}^{0} \lesssim \epsilon^{n}, \\
\delta_{3} \simeq \frac{1}{16 \pi^{2}}\left[\left(\lambda_{\tau}^{2}+4 \epsilon^{4 k+2 k^{\prime}}\right) \delta_{3}^{0}+2 \epsilon^{4 k+2 k^{\prime}+n}\right] \ln \frac{M_{\mathcal{N}}}{M_{X}}+\delta_{3}^{0} \lesssim \epsilon^{n}, \\
\Delta_{1} \simeq \Delta_{1}^{0}+\frac{1}{16 \pi^{2}}\left[\left(2 \epsilon^{4 k+2 k^{\prime}}-\epsilon^{2 n-2 k^{\prime}}\right) \Delta_{1}^{0}+6 \epsilon^{2 k+2 n}\right] \ln \frac{M_{\mathcal{N}}}{M_{X}}, \\
\Delta_{2} \simeq \frac{3}{8 \pi^{2}} \epsilon^{2 k+2 n} \ln \frac{M_{\mathcal{N}}}{M_{X}},
\end{gathered}
$$

where $\delta^{0}, \Delta^{0}$ denote their values at $M_{X}$, while $\delta, \Delta$ in (60)-(63) are their values at $M_{\mathcal{N}}$ (at $M_{X}, \Delta_{2}^{0}=0$ due to $\mathcal{U}(1)$ ). Equations (60)-(63) convince us that down to scale $M_{\mathcal{N}}$, the conditions (54), (55) are easily satisfied even for $M_{X} / M_{\mathcal{N}} \sim 10^{15}$.

At scale $M_{\mathcal{N}}$ the states $\mathcal{N}_{1,2}$ decouple and $\hat{M}_{\nu}$ is generated. Therefore, below $M_{\mathcal{N}}$, $\hat{M}_{\nu}$ will run through appropriate $d=5$ operators. The RGE for $\hat{M}_{\nu}$ is:

$$
16 \pi^{2} \frac{d}{d t} \hat{M}_{\nu}=-\bar{c}_{a} g_{a}^{2} \hat{M}_{\nu}+\hat{\lambda}_{e} \hat{\lambda}_{e}^{T} \hat{M}_{\nu}+\hat{M}_{\nu} \hat{\lambda}_{e} \hat{\lambda}_{e}^{T}+6 \operatorname{tr}\left(\lambda_{u}^{T} \lambda_{u}\right) \hat{M}_{\nu},
$$

where $\bar{c}_{a}=\left(\frac{6}{5}, 6,0\right)$.

The important point is that in (37) the elements $(1,1)(2,3),(3,3),(2,2) \lesssim m \epsilon^{n}$. Using the notations 


$$
\frac{M_{\nu}^{11}}{M_{\nu}^{12}}=X_{1}, \frac{M_{\nu}^{22}}{M_{\nu}^{12}}=X_{2}, \quad \frac{M_{\nu}^{33}}{M_{\nu}^{12}}=X_{3}, \quad \frac{M_{\nu}^{23}}{M_{\nu}^{12}}=X_{4}
$$

the conditions

$$
X_{i} \lesssim \epsilon^{n}
$$

must be satisfied at scale $M_{Z}$. If in (65) $M_{\nu}^{12}$ will be replaced by $M_{\nu}^{13}$, the conditions (66) will still occur, since we require that $M_{\nu}^{12} \sim M_{\nu}^{13}$. Let us first demonstrate that the ratio $M_{\nu}^{12} / M_{\nu}^{13} \equiv r$ does not change significantly under renormalization. Using (64), the RGE for $r$ :

$$
16 \pi^{2} \frac{d \ln r}{d t} \simeq-\lambda_{\tau}^{2}
$$

with the approximate solution

$$
r \simeq r^{0}\left(1-\frac{\lambda_{\tau}^{2}}{16 \pi^{2}} \ln \frac{M_{Z}}{M_{\mathcal{N}}}\right) \sim r^{0}
$$

where $r$ and $r^{0}$ are the values at scales $M_{\mathcal{N}}$ and $M_{Z}$ respectively. (68) demonstrates that the magnitude of $r$ is not significantly altered. The RGEs for $X_{i}$, to good approximations, are:

$$
\begin{aligned}
& 16 \pi^{2} \frac{d \ln X_{1}}{d t} \simeq-\lambda_{\mu}^{2}, \quad 16 \pi^{2} \frac{d \ln X_{2}}{d t} \simeq \lambda_{\mu}^{2}, \\
& 16 \pi^{2} \frac{d \ln X_{3}}{d t} \simeq 2 \lambda_{\tau}^{2}, \quad 16 \pi^{2} \frac{d \ln X_{4}}{d t} \simeq \lambda_{\tau}^{2},
\end{aligned}
$$

with approximate solutions

$$
\begin{gathered}
X_{1(2)} \simeq X_{1(2)}^{0}\left(1 \mp \frac{\lambda_{\mu}^{2}}{16 \pi^{2}} \ln \frac{M_{Z}}{M_{\mathcal{N}}}\right) \simeq X_{1(2)}^{0}, \\
X_{3} \simeq X_{3}^{0}\left(1+\frac{\lambda_{\tau}^{2}}{8 \pi^{2}} \ln \frac{M_{Z}}{M_{\mathcal{N}}}\right) \sim X_{3}^{0}, \quad X_{4} \simeq X_{4}^{0}\left(1+\frac{\lambda_{\tau}^{2}}{16 \pi^{2}} \ln \frac{M_{Z}}{M_{\mathcal{N}}}\right) \sim X_{4}^{0},
\end{gathered}
$$

which demonstrates that the conditions in (66) are satisfied since $X_{i}$ is proportional to $X_{i}^{0}$, which does not exceed $\epsilon^{n}$.

We therefore can conclude that renormalization effects do not significantly affect $\hat{M}_{\nu}$ which has the desired form (37) at scale $M_{Z}$. Let us note that the papers in [25] investigate the influence of renormalizations on neutrino mixings and oscillations, while [26] present models with large neutrino mixings, that are stable against radiative corrections. 
In conclusion, within MSSM and beyond we have addressed the problem of flavor and neutrino anomalies. For a simultaneous resolution an anomalous $\mathcal{U}(1)$ flavor symmetry was invoked. Bi-maximal neutrino mixing texture was generated and the observed hierarchies between charged fermion masses and their mixings were obtained. Renormalization group analysis shows that the bi-maximal neutrino mixing picture is stable against quantum corrections.

Z.T. would like to thank the Organizers of NATO 2000 meeting for warm hospitality at Cascais - Portugal and for their support.

\section{References}

[1] Y. Fukuda et al., Phys. Rev. Lett. 82 (1999) 2644; Phys. Lett. B 467 (1999) 185.

[2] J. Bahcall, P. Krastev, A. Smirnov, hep-ph/0006078; J. Bahcall, hep-ex/0002018.

[3] C. Athanassapoulos et al., Phys. Rev. Lett. 75 (1995) 2650; 77 (1996) 3082; nuclex/9706006.

[4] E. Akhmedov, hep-ph/0011136, hep-ph/0011353; See also referances therein.

[5] M. Gell-Mann, P. Ramond and R. Slansky, in: Supergravity, eds. P. van Nieuwenhuizen and D. Freedman (North-Holland, Amsterdam, 1979) p. 315; T. Yanagida, Prog. Th. Phys. B 135 (1979) 66. R. Mohapatra and G. Senjanovic, Phys. Rev. Lett. 44 (1980) 912.

[6] R. Barbieri at el, hep-ph/9807235.

[7] A.S. Joshipura, S.D. Rindani, hep-ph/9811252; R.N. Mohapatra, A. Perez-Lorenzana and C.A. de S. Pires, hep-ph/9911395.

[8] Q. Shafi and Z. Tavartkiladze, hep-ph/0002150.

[9] Y. Nir, hep-ph/0002168; N.F. Bell, R.R. Volkas, hep-ph/0008177; T. Kitabayashi, M. Yasue, hep-ph/0011153.

[10] M. Green and J. Schwarz, Phys. Lett. B 149 (1984) 117.

[11] M. Dine, N. Seiberg and E. Witten, Nucl. Phys. B 289 (1987) 584; J. Atick, L. Dixon and A. Sen, Nucl. Phys. B 292 (1987) 109; M. Dine, I. Ichinose and N. Seiberg, Nucl. Phys. B 293 (1987) 253. 
[12] L. Ibañez, G.G. Ross, Phys. Lett. B 332 (1994) 100; P. Binetruy, P. Ramond, Phys. Lett. B 350 (1995) 49; V. Jain, R. Shrock, Phys. Lett. B 352 (1995) 83; E. Dudas, S. Pokorski, C. Savoy, Phys. Lett. B 369 (1995) 255; K. Choi, E.J. Chun, H. Kim, Phys. Lett. B 394 (1997) 89; Z. Berezhiani, Z. Tavartkiladze, Phys. Lett. B 396 (1997) 150; B 409 (1997) 220; N. Irges, S. Lavignac, P. Ramond, Phys. Rev. D 58 (1998) 035003; Q. Shafi, Z. Tavartkiladze, hep-ph/9904249; hep-ph/9905202.

[13] P. Binetruy , S. Lavignac, S. Petcov, Nucl. Phys. B 496 (1997) 3; G. Leontaris, N. Tracas, Phys.Lett. B 431 (1998) 90; R. Barbieri et al., JHEP 9812 (1998) 017; Y. Grossman, Y. Nir, Y. Shadmi , JHEP 9810 (1998) 007; M. Fukugita, M. Tanimoto, T. Yanagida, hep-ph/9809554; M. Gomez et al., Phys. Rev. D 59 (1999) 116009; C. Froggat, M. Gibson, H. Nielsen, hep-ph/9811265; S. Kang, C. Kim, hep-ph/9811379; R. Mohapatra, hep-ph/9910365; J. Feng, Y. Nir, hep-ph/9911370; G. Altarelli, F. Feruglio, I. Masina, hep-ph/0007254. M. Berger, K. Siyeon, hep-ph/0010245.

[14] P. Ramond, hep-ph/9808489, and references therein; J. Sato and T. Yanagida, hepph/9809307; See also F. Vissani, hep-ph/9810435.

[15] D. Suematsu, Phys. Lett. B 392 (1997) 413; S. Davidson, S.F. King, hep-ph/9808296.

[16] Q. Shafi and Z. Tavartkiladze, Phys. Lett. B 451 (1999) 129.

[17] P. Binetruy et al., Nucl. Phys. B 496 (1997) 3; V. Barger, T.J. Weiler and K. Whisnant, Phys. Lett. B 427 (1998) 97; Y. Grossman, Y. Nir and Y. Shadmi, hep-ph/9808355; Q. Shafi and Z. Tavartkiladze, Phys. Lett. B 448 (1999) 46, hepph/9811463. In the context of realistic $S U(4)_{c} \times S U(2)_{L} \times S U(2)_{R}$ model with maximal $\nu_{\mu}-\nu_{\tau}$ mixing, it was also discussed in ref. [19].

[18] Q. Shafi and Z. Tavartkiladze, Phys. Lett. B 487 (2000) 145.

[19] Q. Shafi and Z. Tavartkiladze, Nucl. Phys. B 549 (1999) 3, hep-ph/9811282.

[20] G. Dvali and A. Pomarol, Phys. Rev. Lett. 77 (1996) 3738; P. Binetruy and E. Dudas, Phys. Lett. B 389 (1996) 503.

[21] M. Dine, A. Kagan, S. Samuel, Phys. Lett. B 243 (1990) 250; S. Dimopoulos, G. Giudice, Phys. Lett. B 357 (1995) 573; N. Arkani-Hamed, H. Murayama, Phys. Rev. D 56 (1997) 6733; J. Hisano, K. Kurosawa, Y. Nomura, Phys. Lett. B 445 (1999) 316; L. Everett et al., Phys. Lett. B 477 (2000) 233; J. Feng, K. Matchev, hep-ph/0011356; See also references therein.

[22] F. Gabbiani, E. Gabrielli, A. Masiero, L. Silvestrini, Nucl.Phys. B477 (1996) 321; G. Eyal, A. Masiero, Y. Nir, L. Silvestrini, hep-ph/9908382. 
[23] A. Nelson, D. Wright, hep-ph/9702359.

[24] Q. Shafi, Z. Tavartkiladze, Phys. Lett. B 473 (2000) 272, hep-ph/9911264.

[25] C. Wetterich, Nucl. Phys. B 187 (1981) 343; P.Chankowski, Z. Pluciennik, Phys. Lett. B 316 (1993) 312; M. Tanimoto, Phys. Lett. B 360 (1995) 41; N. Haba at el., hep-ph/9810471; J. Ellis, S. Lola, hep-ph/9904279; P. Chankowski, W. Krolikowski, S. Pokorski, hep-ph/9910231.

[26] Y. Wu, hep-ph/9905222; S. Lola, hep-ph/0005093; T. Miura at el., hep-ph/0005267;

S. King, N. Singh, Nucl. Phys. B 591 (2000) 3; E. Akhmedov at el., hep-ph/0008010; K. Balaji at el., hep-ph/0011263. 